\title{
Design of Campus Comprehensive Service System Based on Android
}

\author{
Han Qin and Wei Xiang* \\ Southwest MinZu University, Chengdu, Sichuan, 610225, P.R. China \\ ${ }^{*}$ Corresponding author
}

\begin{abstract}
In this paper, we design a mobile terminal campus comprehensive service system, which based on Android, and combines HTML, PHP scripting language programming, Java object-oriented programming, as well as using Phonegap open source framework, and use the MySQL user database design. From function, form a comprehensive service system integrating new plate, life plate and learning plate, convenient to college students in the practical application of all kinds of information query and similar interests together.
\end{abstract}

Keywords-android; the mobile terminal; campus integrated service system

\section{INTRODUCTION}

With the rapid development of science and technology, the function of mobile phones is no longer just for the convenience of contacting people, more and more people use it to entertain or work. The reason why smart phones can fit into our lives in a short time is because of the launch of the android operating system and the full application of 4G technology and wireless network. Android ${ }^{[1]}$ is a set of the real meaning of open platform for mobile devices integrated, with the birth of 3G network, mobile terminal is no longer the terminal of communication network, but the emergence of $4 \mathrm{G}$ network makes it the terminal of Internet.The application of the intelligent mobile terminal have very big development space, and college students are the larger group of intelligent end users, therefore, there are some mobile applications for college students, but the function is not comprehensive enough, so it cannot be combined closely with the resources of colleges and the students' life and learning to provide good service for the students, therefore, developing a comprehensive campus service system [2] based on Android is of great significance ${ }^{[3]}$, which will solve these problems well.

\section{B/S STRUCTURE}

$\mathrm{B} / \mathrm{S}$ structure ${ }^{[4]}$ is an improved structure of $\mathrm{C} / \mathrm{S}$ structure with the rise of web technology. Based on the C/S structure, it mainly simplifies the client load, centralizes the core part of the system function to the server side, and realizes the crossplatform access to the computer and various resources on the network. The B/S structure can be divided into three units: presentation layer (Web Browser), functional layer (Web Server with application extension function), data layer (database Server). In the Browser/Server system, the user can send a request to the server side of the network through the client browser, the server receives and processes the request, and then returns the data requested by the user (web file, picture, sound, etc.) to the browser.

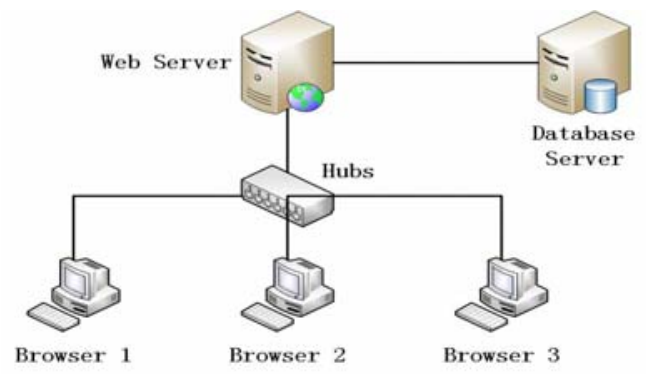

FIGURE I. B/S STRUCTURE

\section{PHONEGAP}

Phonegap is an open-source development framework for Adobe, using HTML, Javascript and CSS technology to develop cross-platform mobile applications. Phonegap supports cross-platform, provides hardware response control and has rich plug-ins to call.

\begin{tabular}{|c|c|c|c|c|c|}
\hline Web & HTML & Javascript & & \multicolumn{2}{|l|}{ CSS } \\
\hline 厉 HTML API & \multicolumn{2}{|l|}{ ת prompt } & \multicolumn{2}{|c|}{ 亿 synchronous $\begin{array}{c}\substack{\text { asynchronous }\\
} \\
\end{array}$} & XHR\&JSOI \\
\hline \multicolumn{6}{|c|}{ HTML Rendering Engine(WebView) } \\
\hline$\Gamma^{\text {HTML Basic Interaction }}$ & $\sqrt{2}$ & \multicolumn{2}{|c|}{ Native Support } & & \\
\hline & Accelerometer & Camera & Media & Compass & \\
\hline & Geolocation & Contacts & Network & File & \\
\hline & Notification & Storage & \multicolumn{2}{|c|}{ Custom Plugins } & \\
\hline \multicolumn{2}{|l|}{ 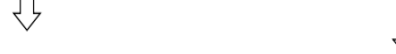 } & \multicolumn{2}{|c|}{ Android API } & \multicolumn{2}{|c|}{ U } \\
\hline \multirow{2}{*}{ Android OS } & Services & \multicolumn{2}{|c|}{ Sensors } & Media & \\
\hline & Input & \multicolumn{2}{|c|}{ Graphics } & ....... & \\
\hline
\end{tabular}

FIGURE II. PHONEGAP STRUCTURE

\section{SySTEM ARCHIRECTURE DESIGN}

\section{A. System Operating Environment}

The entire system works with the front-end Android client and the back-end including the web server and the database server. WEB server is configured with open source free 
Apache2.4 and Tomcat7.0 server integration, Apache only supports static pages such as HTML, but it is fast and efficient, while Tomcat can run JSP pages and Servlet other than HTML, a combination of these make the system run in a good environment, enhances the working efficiency of the system, The system web database data store uses MySQL database.

\section{B. System Framework ${ }^{[5]}$}

When users in the use, first from a static HTML page, a web browser response to a user action, through HTTP requests the user requests to the web server, the server to its operating data processing, This process includes the JSP initialization model as the controller and calls the JSP as the view, JavaBeans performs the corresponding business logic processing capabilities based on the user's actions through the interface, simultaneously calling the data from the database and storing the processing results into the database, and uploading the information to the JSP page.This process is the data interaction between the server and the database, and then the HTTP response is finally interpreted by the Web server, and the server processing result is returned to the user terminal, which enables the user to get the required information or data.

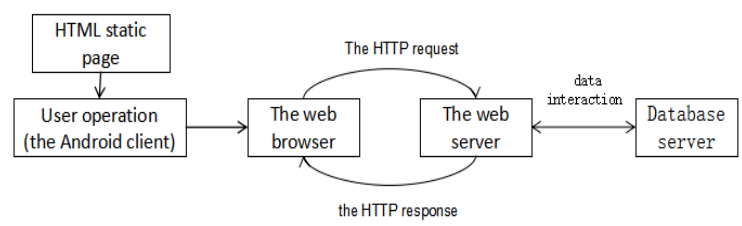

FIGURE III. SYSTEM FRAME DIAGRAM

\section{System Function Design}

1) System client function analysis: The system is divided into three functional sections from the point of view of the users. They are the freshmen who help freshmen integrate into college life more quickly, and the life and learning sections for all the students in the university. Basic covers all the campus information that students need on the Internet.

a) The new plate: It includes student cart instructions, reporting process, map and transportation, campus profile, campus network and school bus schedule. In map and transportation, the map and navigation are called by accessing the autonavi map SDK, to facilitate journey; The campus network illustrates the usage and billing rules of the Intranet, so that students can easily surf the Internet on campus. The school bus will be run on the school bus schedule at regular intervals to facilitate teachers and students to arrange the trip.

b) Life plate: It includes club introduction, second-hand trading platform, communication platform and part-time platform. Among them, Second-hand trading platform ${ }^{[6]}$ can make some idle articles be digested inside the campus. Since the scope is small, it can be seen in real life, so it is convenient to trade. Exchange BBS to allow groups of common interest to communicate or organize various activities; Part-time information will be posted on the part-time platform, including the location of the work and the specific salary. c) The learning plate: It includes mutual excitation platform, optional course notes, examination inquiry, library and schedule. Among them, the mutual excitation platform is similar to the small learning BBS, which is divided into four and six levels, the entrance examination area and the examination area of various certificates; There will be some attentions and procedures in the course selection. The library module not only has the function of the online library, but also shows the number of people in the library in real time, and avoids the embarrassing situation where the library has no location. You can choose to add courses manually or to load the schedule directly according to the educational system.

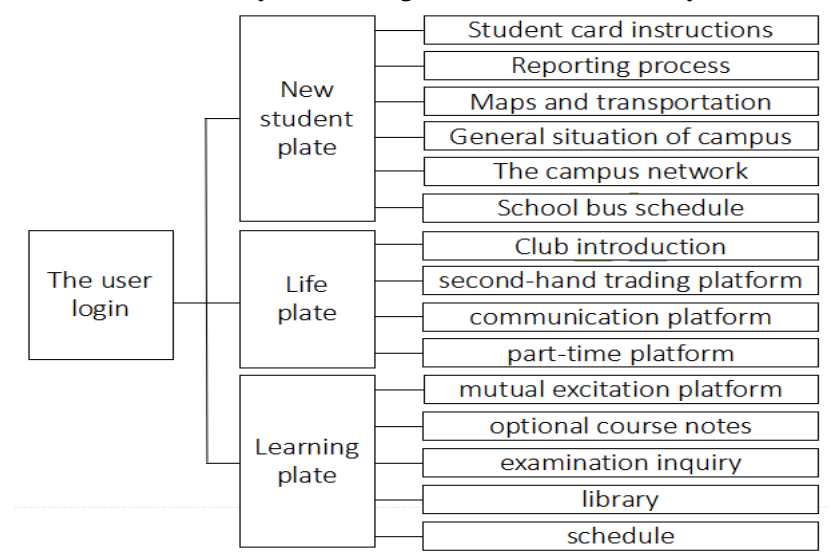

FIGURE IV. SYSTEM CLIENT FUNCTION DISTRIBUTION DIAGRAM

\section{2) System server function analysis}

Android client uses the integrated development tool Android Studio to develop. The Web server is developed with PHP, mainly considering the high efficiency of PHP, strong cross-platform, and perfect cooperation with MySQL.

The front-end Android client is mainly responsible for user login and campus information, class schedule query, performance query and other information query plates. Users in the Android client after a successful login, click on the corresponding query function button, at this time the request, Via WiFi or $4 \mathrm{G}$ wireless network will command is passed to the backend web server, database and query the background, then the query results returned in the JSON data format, using the Android UI design should be displayed in a grid view to the client.

Learning and live communication module mainly divided into four parts, respectively is second-hand trading platform and communication platform, mutual excited platform and part-time platform, the four platforms from different functions to achieve the exchange of information between users, Through the intermediary role of the server, users can communicate directly on the client side, so that students, teachers and parents can communicate effectively through the network. 


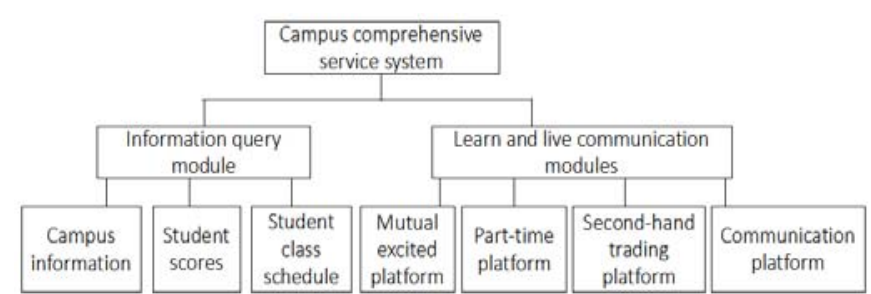

FIGURE V. SYSTEM SERVER FUNCTION DISTRIBUTION DIAGRAM

\section{Database Design}

Database design ${ }^{[7]}$ is for a given application environment, constructing the optimal database model, establish a database and application systems, to store data effectively and meet the application requirements of various users (information requirements and processing requirements).

Based on the system function analysis, for the user to have good experience and query to the appropriate useful information, the system database will mainly establish student information table, student performance table, corporate information table, course inquiry form and so on.

\section{CONCLUSION}

This system is mainly based on the Android system, which adopts the open source framework of Phonegap and the MySQL database, and interacts with users through the web server. Android platform to promote the technology innovation, and developers can very convenient to design the characteristic products, including the comprehensive service system for students' campus life service, which is close to the user's life, making the technology better serve for the students, and has great market development potential.

\section{ACKNOWLEDGEMENT}

This work was partially supported by Sichuan Youth Science and Technology Innovation Research Team (2017TD0028).Also was supported by the Fundamental Research Funds for Central University, Southwest Minzu University (2017NZYQN45).

\section{REFERENCES}

[1] Lv Wei. Design and implementation of mobile learning platform based on Android [D]. Nanjing: nanjing normal university, 2013.

[2] Lei Yun et al. Design and discussion of teaching examples in the experimental course of android application development. Test weekly. Zhejiang: lishui college, 2012.

[3] Wei Wei. Analysis on the promotion and application strategy of digital campus in universities. China science and education innovation guide. Beijing: China university of geosciences, 2011.

[4] Zhao Feng, Zhao DuanZheng. Based on B/S,C/S integration mode application software development research. China's science and technology information.

[5] Li RiDian. Design and implementation of the framework for the development of information Android applications. Beijing: Beijing university of posts and telecommunications.2015.

[6] Lv XiaoMeng, Gao LianTing, etc. On the idea of building a second-hand commodity trading platform in university campus. Foreign trade and economic cooperation. Shenyang, dalian: shenyang university of aeronautics and astronautics, dalian university of technology.2016.

[7] Xiong Hui. Practical exploration of database design theory in software development. Wireless Internet technology. Sichuan: sichuan vocational and technical college.2017.P58-59. 